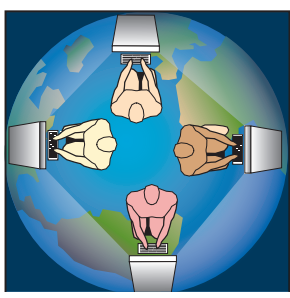

\title{
Resolving Barriers to Licensure Portability for Telerehabilitation Professionals
}

\author{
Ellen R. Cohn, PhD, Janice A. Brannon, MA, Jana Cason, DHS, OTR/L \\ American Telemedicine Association: Telerehabilitation Special Interest Group, \\ Licensure Portability Working Group, Report
}

\section{Introduction}

Telerehabilitation (TR) refers to the use of information and communication technologies to deliver rehabilitation services. ${ }^{1}$ As per the conventions of the American Telemedicine Association's Special Interest Group on Telerehabilitation, ${ }^{2}$ the term 'clients' hereafter refers to recipients of telerehabilitation services -- and is inclusive of patients in medical and non-medical (e.g., school, home, etc.) settings.

\section{Challenges}

Rehabilitation professionals (e.g., audiologists, occupational therapists, physical therapists, speech-language pathologists, etc.) may only engage in telerehabilitation in states in which they hold a professional license. This is akin to needing a different driver's license to drive in every US state and territory! Clinicians report duplicative paperwork, expense, and wait times; for some professions, there can be lack of uniformity for state credentialing requirements and fees.

With the changing health care system, attention needs to be paid to easing the ability of clinicians to obtain license or recognition in multiple states for the purpose of providing telerehabilitation services.

The 2007 Second Report to the State Alliance for E-Health identified a key challenge: "The current credential verification method is a very time-consuming, paper-based process for state boards that contributes to the reluctance of healthcare professionals to apply for multiple licenses" and advanced a solution: "The Taskforce believes that state boards can reduce these timeframes by establishing a centrally coordinated credentials verification organization for each profession to conduct the primary source verification of applicants' credentials. In order to facilitate the collection of credentialing data for this system and ensure the portability of these credentials, state boards should collaborate to develop a nationwide core set of credentialing requirements that their respective health professionals would have to meet in order to obtain a license"3 (p.6).

The challenges of licensure and the changing health care delivery system are such that the US Federal Communications Commission urged state licensing boards to accommodate multi-state licensure, concluding: "If states fail to develop reasonable e-care licensing policies by the next 18 months [by September, 2011] Congress should consider intervening to ensure Medicare and Medicaid beneficiaries are not denied the benefits of e-care." 4

In addition, the Health Resources and Services Administration addressed the issue, urging licensure boards to design methods to facilitate portability of credentials across state lines. Some healthcare professionals such as physicians and nurses have already made strides toward establishing inter-state licensing mechanisms; the nurses through the use of state compacts and physicians through the use of expedited licensure. All healthcare professional licensure boards have been urged to design methods to facilitate portability of credentials across state lines. ${ }^{3}$

\section{Impact on Consumers and States}

Licensure portability would solve some important problems including:

- Access: Clients in many underserved areas do not have access to rehabilitation services whether due to economic, geographic, or impaired mobility issues. Telerehabilitation could help make services more accessible.

- Shortages \& Suboptimal Distribution of Providers: Healthcare providers and specialists (both individual and team based) may not be equitably distributed geographically. Mobility and availability may be improved through the use of telerehabilitation.

- Administrative Redundancy: Many state systems are supporting expensive and duplicative licensing practices. 


\section{Potential Approaches}

There are several alternative models of licensure portability:

1. Mutual Recognition Compacts - similar to a driver's license and is based on the provider having one full license in their home state but can practice in other states that have entered into a legal agreement with that state.

2. Expedited License- utilizes core standards, a uniform application and credential verification that can be stored and sent to multiple states where licensure is desired.

3. Limited License - a license granted by each state that is limited in scope to the practice of telehealth across state lines. This is in addition to the provider having at least one full license in their home state.

4. National License- based on a universal standard for each profession and administered at the state or national level

5. Federal Pre-emption - the federal government pre-empts state statutes and would grant licensure for inter-state practice for programs paid for by federal dollars.

Recognizing common denominators to all portability models, the US Department of Health and Human Services, Health Services and Resources Administration, Health Licensing Board Report to Congress (Requested by Senate Report 111-66) identified three key enablers to facilitate licensure portability: "uniform core licensure requirements; common/uniform license application; and credential verification organizations." 5

In 2010, the Special Interest Group on Telerehabilitation of the American Telemedicine Association (ATA) convened an interdisciplinary working group (WG) with the goal of educating stakeholders regarding the barriers to interstate telerehabilitation and to seek new models that can extend the benefits of an electronic, multi-state practice.

The WG is exploring these models and ways to establish the key enablers as a means to facilitate licensure portability among the rehabilitation professions.

\section{Benefits of Inter-State Licensure Portability}

- Clients will have access to the specialist that they need regardless of state boundaries.

- State governments could reduce duplicative processes and costs (i.e., reallocation without relocation).

- Clinicians will incur reduced fees and faster processing times.

- State professional practice boards could reallocate resources to focus on enforcement and complex problems.

- Rehabilitation professionals who currently engage in telerehabilitation across state lines without secondary state licenses will be subject to greater oversight, to the benefit of consumers.

- Both clients and providers will benefit from time efficiencies and reduced travel.

- Increased client compliance, motivation, and therapeutic outcomes may be achieved.

\section{Conclusions}

The current state-based licensure and regulation of rehabilitation professionals does not facilitate the practice of telerehabilitation across state lines. Given today's equipment capabilities and consumer adoption of the electronic delivery of many kinds of services, health care providers, including rehabilitation professionals, should be able to serve clients wherever they are needed. 


\section{Acknowledgements}

This document was authored by a sub-committee of the ATA Telerehabilitation Special Interest Group (SIG), with the generous input of members of an interdisciplinary working group (WG). It is not an official document of any professional association, federation or association of state licensing boards. For information: Ellen Cohn, ecohn@pitt.edu, Chair, Telerehabilitation SIG of the American Telemedicine Association. Graphic support was supplied by the RERC on Telerehabilitation funded by NIDRR Department of Education, Washington DC, Grant \#H133E090002.

\section{Endnotes:}

1. The terminology used to describe such delivery varies (e.g., "telerehabilitation," "telemedicine," "telehealth," "telepractice," and "e-health").

2. Telerehabilitation Special Interest Group on Telerehabilitation, A Blueprint of Telerehabilitation Guidelines, http://www. americantelemed.org/i4a/pages/index.cfm?pageID=3311, or: doi: 10.5195/ijt.2010.6063.

3. Second Report from the Health Care Practice Taskforce to the State Alliance for E-Health, October 3, 2007, financed by funds from the US Department of Health and Human Services, Office of the National Coordinator for Health IT (ONCHIT) under a contract with the National Governors Association for the State Alliance for e-Health." http://www.nga.org/files/live/sites/NGA/ files/pdf/0710EHEALTHHCPREPORT.PDF

4. Connecting America: The National Broadband Plan, http:// www.broadband.gov/plan/, released on March 17, 2010.

5. www.hrsa.gov/ruralhealth/about/telehealth/licenserpt10.pdf 
